\title{
Professor Dr. med. Paul Ernst Robert, 1906-1953
}

Die schweizerische Dermatologie ist durch den Verlust eines ihrer Besten in tiefe Trauer versetzt worden. Der Vorsteher der Dermato-logischen Universitätsklinik Bern, Professor Dr. Paul Robert, ist in der Frühe des 7. August, in Schönried, unmittelbar vor der Heimreise aus den Ferien, plötzlich und unerwartet durch eine Herzkrise mitten aus einer glänzenden Laufbahn abberufen worden.

Nach seinem Staatsexamen im Frühjahr 1930 in Zurich hatte er zunächst je ein Jahr am Pathologisch-anatomischen Institut unter Professor von Meyenburg, am Hygienischen Institut unter Professor Silberschmidt, sowie an der Chirurgischen Klinik unter Professor Clairmont, und hernach während dreier Jahre an der Dermatologi-

Professor Dr. med. Paul Ernst Robert

19061953

Die schweizerische Dermatologie ist durch den Verlust eines ihrer Besten in tiefe Trauer versetzt worden. Der Vorsteher der Dermato-logischen Universitätsklinik Bern, Professor Dr. Paul Robert, ist in der Frühe des 7. August, in Schönried, unmittelbar vor der Heimreise aus den Ferien, plötzlich und unerwartet durch eine Herzkrise mitten aus einer glänzenden Laufbahn abberufen worden.

Nach seinem Staatsexamen im Frühjahr 1930 in Zurich hatte er zunächst je ein Jahr am Pathologisch-anatomischen Institut unter Professor von Meyenburg, am Hygienischen Institut unter Professor Silberschmidt, sowie an der Chirurgischen Klinik unter Professor Clairmont, und hernach während dreier Jahre an der Dermatologi-

$1 / 8 . .3 / 8 \cdot 3 / 8 / 8 \grave{a}^{*}$

Permatologioa, Vol. 107, No. 3 (1953)

11

162 Prof. Dr. med. Paul Ernst Robert

schen Klinik unter Professor Miescher als Assistent gearbeitet. Im November 1936 siedelte er zur internistischen Ausbildung unter den Herren Professoren Rudolf Staehelin und Massini nach Basel über und wandte sich im Herbst 1937 durch Ubernahme der freiwerdenden Oberarztstelle an der dortigen Dermatologischen Klinik wiederum der Dermatologie zu. Im Frühjahr 1941 reichte er der medizinischen Fakultät Basel seine Habilitationsarbeit ein und erlebte, während sein Gesuch um Erteilung der venia legendi nocb in Behandlung war, die stolze Freude, von der Fakultät der Universität Bern, die - auf der Suche nach einem Vertreter fur das Fach der Dermatologie und Venereologie - infolge seiner sehr vielseitigen Ausbildung auf Robert aufmerksam geworden war, der Berner Regierung zur Berufung vor-geschlagen und von dieser berufen zu werden. In raschem Lauf hatte er somit im Alter von erst 35 Jahren ein hohes Ziel erreicht. Die schweizerische Wissenschaft ist der Fakultät und den Behörden Berns zu größtem Dank verpflichtet, daß sie dem noch jugendlichen For-scher ihr Vertrauen schenkten. Er hat sich dessen auch würdig erwiesen. Trotzdem er anfänglich mit organisatorischen und administrativen Arbeiten sehr belastet war, hat er sich neben der gewissenhaftesten Führung der Klinik und der sorgfältigsten Betreuung des Unterrichts, den er in geistreicher Weise 
hervorragend lebendig zu gestalten wußte, sofort auf das in-tensivste wissenschaftlichen Forschungsaufgaben zugewendet und auch Mitarbeiter und Schüler lebhaft dazu angehalten. Als besonders von ihm gepflegte Gebiete seien hier nur genannt die Beziehungen der Hautkrankheiten zu internen Vorgängen und Stö-rungen, insbesondere des Stoffwechsels, und die Probleme der Pig-mentbildung und deren Anomalien. Seine Studien und die Arbeiten seiner Mitarbeiter sind noch zu frisch in der Erinnerung, als daß auf sie, die meist auch in dieser Zeitschrift publiziert worden sind, noch-mals ausführlicher eingegangen werden müßte. Alle Arbeiten zeichnen sich in gleicher Weise durch klare Fragestellung und übersichtlichen Aufbau, durch äußerste Genauigkeit in der Methodik und der For-mulierung, durch kritische, vorsichtig abwägende Interpretation der Resultate und durch eine eminente Kenntnis und Beherrschung der Literatur aus.

Robert hat mit seinen Resultaten Vieles und Wesentliches zur Förderung und Mehrung unserer Kenntnisse beigetragen. Wissen-schaftliches Forschen war ihm Lebensnotwendigkeit. Es war ihm ein innerstes Bedürfnis und tiefstes Anliegen, immer wieder von neuem

Prof. Dr. med. Paul Ernst Robert

163

zu versuchen, weitere Einblicke in die biologischen Vorgänge im Innern des Körpers und in der Haut zu gewinnen und ihre Bedeutung zu verstehen, mit dem Ziel, immer noch bessere und sicherere Hilfs-mittel zur Behandlung seiner Kranken zu finden. Hiefür beseelte ihn ein ungeheurer, fast fanatisch zu nennender Arbeitswille, hinter dem alles, auch seine Gesundheit, zurücktreten mußte.

Viele seiner Arbeiten hat er zuerst in den Sitzungen der Schweizeri-schen Gesellschaft für Dermatologie vorgetragen. Er war der Gesell-schaft dadurch ein äußerst anregendes und eifriges Mitglied, das mit seinen ideenreichen Äußerungen und geistsprühenden Diskussionsbemerkungen an unseren Tagungen außerordentlich belebend wirkte. Vielleicht klangen die letzteren manchmal etwas scharf oder sar-kastisch, man konnte und durfte aber deswegen nicht mit ihm rech-ten. Vielleicht mochte schon hie und da auch einmal eine gewisse Sensitivität gegenüber einer Kritik an von ihm vertretenen Auffas-sungen mitspielen, seine Voten entsprangen jedoch immer lauteren Quellen, nämlich seiner äußersten Gewissenhaftigkeit in alien wissen-schaftlichen Fragen, seiner philosophischen Bescheidenheit vor den Geheimnissen biologischen Geschehens und seinem Wissen um die Relativität unserer Kenntnisse, Eigenschaften, die er in sich be-wahrte, über die er kaum je sprach, wie auch wohl nur ganz wenige wissen dürften, daß er ungewöhnlich musikalisch veranlagt war.

Es liegt eine große Tragik darin, daß dieser hervorragende und unermüdliche Arbeiter so mitten aus seinem Planen und Arbeiten herausgerissen worden ist. Er hat versucht, ein Maximum an Leistun-gen zu erzielen und sich dabei wohl selbst verzehrt. Er hinterläßt eine Lücke, die in seiner Art nicht ersetzt werden kann und wird von alien, die ihn gekannt haben, auf das schmerzíichste vermißt werden.

W. Lutz, Basel 